\section{Intrinsische Schlafstörungen}

Helga Peter ${ }^{1}$ und Thomas Penzel ${ }^{2}$

${ }^{1}$ Marburg, Deutschland

${ }^{2}$ Interdisziplinäres Schlafmedizinisches Zentrum, Charité Universitätsmedizin Berlin, Berlin, Deutschland

\section{Englischer Begriff}

intrinsic sleep disorders

\section{Definition}

Schlafstörungen bzw. schlafmedizinische Krankheiten aus von innen her wirkender Ursache. Die Bezeichnung stand in der ersten Version der Internationalen Klassifikation der Schlafstörungen (ICSD) von 1990 für eine von drei Kategorien von Schlafstörungen innerhalb der Hauptkategorie der > „Dyssomnien“. Die „ „ICSD-2“ von 2005 hat diese Einteilung verlassen. Siehe dazu auch $\triangleright$,Diagnostische Klassifikationssysteme“. 